\title{
Modified Synchronization Analysis of Complex System
}

\author{
Xuefeng Liang \\ School of Mathematic and statistics, Tianshui Normal University, Tianshui, 741001 \\ liangxuefeng@163.com
}

\begin{abstract}
This paper studies the modified projective synchronization of complex system, focusing on fractional-order hyper-chaotic nonlinear system. Based on the stability theory of nonlinear system and control method, effective scheme of synchronization for complex system is designed. Numerical simulations results showed that the proposed scheme is effectiv and feasible.
\end{abstract}

Keywords: Complex system, Synchronization, Nonlinear control

\section{Introduction}

In recent years, many researchers have focused their attention on thyperchaotic systems in many fields [1,2]. Also, Fractional calculus has considered as the generalization of the conventional calculus. It can be seen that the fractional calculus has applications in different fields, such as chaos synchronization, secure communication [3]. Moreover, complex dynamic behaviors including bifurcations and chaos exist in fractional-order systems [4].

Recently, many researchers investigated the dynamics of chaotic systems with complex variables. Modified projective synchronization (MPS) of systems in real space has been investigated are extended to synchronize fractional-order chaotic complex systems [5,6]. Motivated by the above discussion, this paper is to study the fractional-order complex system.

\section{Synchronization scheme}

In this section, an scheme is presented via the idea of nonlinear technique and the fractional-order stability theory to study the MPS of two identical fractional-order complex nonlinear systems. Suppose the derivative order $q_{1}=q_{2}=q_{3}=q_{4}=q$.

The fractional-order complex drive as follows:

$$
D_{*}^{q} y=f(y),
$$

The response system after coupling is given by:

$$
D_{*}^{q} z=f(z)+P(z, g)
$$

where $q \in(0,1]$ is the derivative order, nonlinear vector functions $f$ is continuous and a goal dynamics $g=M y=\left[m_{1} y_{1}, m_{2} y_{2}, \ldots, m_{n} y_{n}\right]^{T}$, where $M=\operatorname{diag}\left(m_{1}, m_{2}, \ldots, m_{n}\right)$ denotes an arbitrary scaling matrix.

The coupling function is obtained as : 


$$
P(z, g)=D_{*}^{q} g-f(g)+\left(H-\frac{\boldsymbol{\Upsilon} f}{\text { \ } g}\right)(z-g),
$$

Define the synchronization error vector as $e=z-g$, the MPS of drive and response systems is achieved if the following condition is satisfied

$$
\lim _{t \rightarrow \infty}\|e\|=\lim _{t \rightarrow \infty}\|z-g\|=0,
$$

Then the error dynamics is described as:

$$
D^{q} e=H e
$$

\section{Simulation results}

The drive system

$$
\left\{\begin{array}{l}
D_{*}^{q_{1}} y_{1}=a\left(y_{1}-y_{2}\right)+y_{4} \\
D_{*}^{q_{2}} y_{2}=b y_{2}-y_{1} y_{3}+y_{4} \\
D_{*}^{q_{3}} y_{3}=\frac{1}{2}\left(\bar{y}_{1} y_{2}+y_{1} \bar{y}_{2}\right)-c y_{3} \\
D_{*}^{q_{4}} y_{4}=\frac{1}{2}\left(\bar{y}_{1} y_{2}+y_{1} \bar{y}_{2}\right)-d y_{4}
\end{array}\right.
$$

and the response system is

$$
\left\{\begin{array}{l}
D_{*}^{q} z_{1}=a\left(z_{1}-z_{2}\right)+z_{4} \\
D_{*}^{q} Z_{2}=b z_{2}-Z_{1} Z_{3}+z_{4} \\
D_{*}^{q} Z_{3}=\frac{1}{2}\left(\bar{z}_{1} Z_{2}+z_{1} \bar{Z}_{2}\right)-c Z_{3}, \\
D_{*}^{q_{4}} Z_{4}=\frac{1}{2}\left(\bar{Z}_{1} Z_{2}+z_{1} \bar{Z}_{2}\right)-d z_{4},
\end{array}\right.
$$

The arbitrary Hurwitz matrix is given by:

$$
\left\{\begin{array}{l}
D_{*}^{q} x_{1}^{\prime}=a\left(x_{3}^{\prime}-x_{1}^{\prime}\right)+x_{6}^{\prime}+m_{1}\left[a\left(x_{3}-x_{1}\right)+x_{6}\right]-a\left(m_{3} x_{3}-m_{1} x_{1}\right)-m_{6} x_{6}+(a-1) e_{1}+a e_{3}-e_{6} \\
D_{*}^{q} \chi_{2}^{\prime}=a\left(x_{4}^{\prime}-x_{2}^{\prime}\right)+x_{6}^{\prime}+m_{2}\left[a\left(x_{4}-x_{2}\right)+x_{6}\right]-a\left(m_{4} x_{4}-m_{2} x_{2}\right)-m_{6} x_{6}+(a-1) e_{2}+a e_{4}-e_{6} \\
D_{*}^{q} x_{3}^{\prime}=b x_{3}^{\prime}-x_{1}^{\prime} x_{5}^{\prime}+x_{6}^{\prime}+m_{3}\left[b x_{3}-x_{1} x_{5}+x_{6}\right]-b m_{3} x_{3}-m_{1} m_{5} x_{1} x_{5}-m_{6} x_{6}+m_{5} x_{5} e_{1}-(1+b) e_{3}+m_{1} x_{1} e_{5}-e_{6} \\
D_{*}^{q} x_{4}^{\prime}=b x_{4}^{\prime}-x_{2}^{\prime} x_{5}^{\prime}+x_{6}^{\prime}+m_{4}\left[b x_{4}-x_{2} x_{5}+x_{6}\right]-b m_{4} x_{4}-m_{2} m_{5} x_{2} x_{5}-m_{6} x_{6}+m_{5} x_{5} e_{2}-(1+b) e_{4}+m_{2} x_{2} e_{5}-e_{6} \\
D_{*}^{q} x_{5}^{\prime}=x_{1}^{\prime} x_{3}^{\prime}+x_{2}^{\prime} x_{4}^{\prime}-c x_{5}^{\prime}+m_{5}\left[x_{1} x_{3}+x_{2} x_{4}-c x_{5}\right]-m_{1} x_{1}\left(m_{3} x_{3}+e_{3}\right)-m_{4} x_{4}\left(m_{2} x_{2}+e_{2}\right)-c m_{5} x_{5}+(c-1) e_{5} \\
D_{*}^{q} x_{6}^{\prime}=x_{1}^{\prime} x_{3}^{\prime}+x_{2}^{\prime} x_{4}^{\prime}-d x_{6}^{\prime}+m_{6}\left[x_{1} x_{3}+x_{2} x_{4}-d x_{6}\right]-m_{1} x_{1}\left(m_{3} x_{3}+e_{3}\right)-m_{4} x_{4}\left(x_{2} m_{2}+e_{2}\right)-d m_{6} x_{6}+(d-1) e_{6} .
\end{array}\right.
$$

The error dynamical system can be rewritten as: 


$$
\left[\begin{array}{l}
D_{*}^{q} e_{1} \\
D_{*}^{q} e_{2} \\
D_{*}^{q} e_{3} \\
D_{*}^{q} e_{4} \\
D_{*}^{q} e_{5} \\
D_{*}^{q} e_{6}
\end{array}\right]=\left[\begin{array}{llllll}
-1 & 0 & 0 & 0 & 0 & 0 \\
0 & -1 & 0 & 0 & 0 & 0 \\
0 & 0 & -1 & 0 & 0 & 0 \\
0 & 0 & 0 & -1 & 0 & 0 \\
0 & 0 & 0 & 0 & -1 & 0 \\
0 & 0 & 0 & 0 & 0 & -1
\end{array}\right]\left[\begin{array}{c}
e_{1} \\
e_{2} \\
e_{3} \\
e_{4} \\
e_{5} \\
e_{6}
\end{array}\right] .
$$

The numerical simulations show that the presented method for the system is effective.
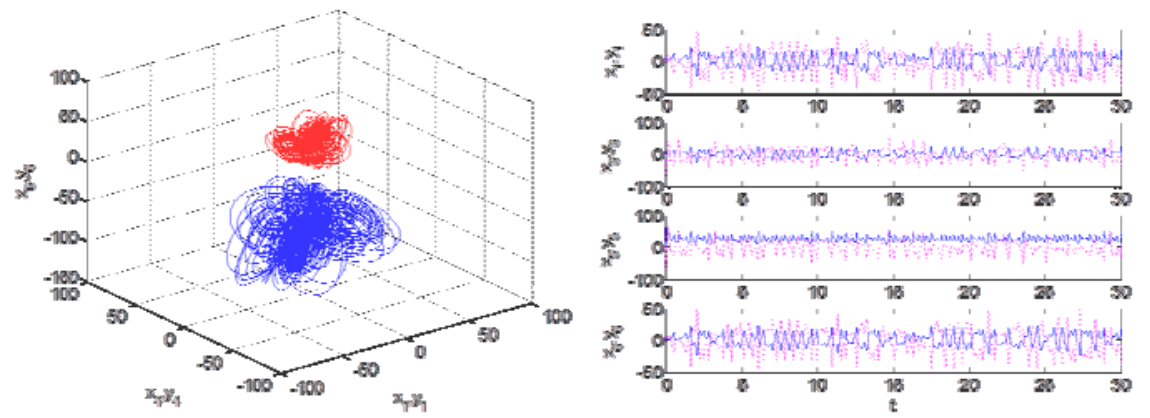

Fig.1. Hyperchaotic attractors and state variables of drive system and response system for scale factors $m_{i}=2(i=1, \ldots, 6)$
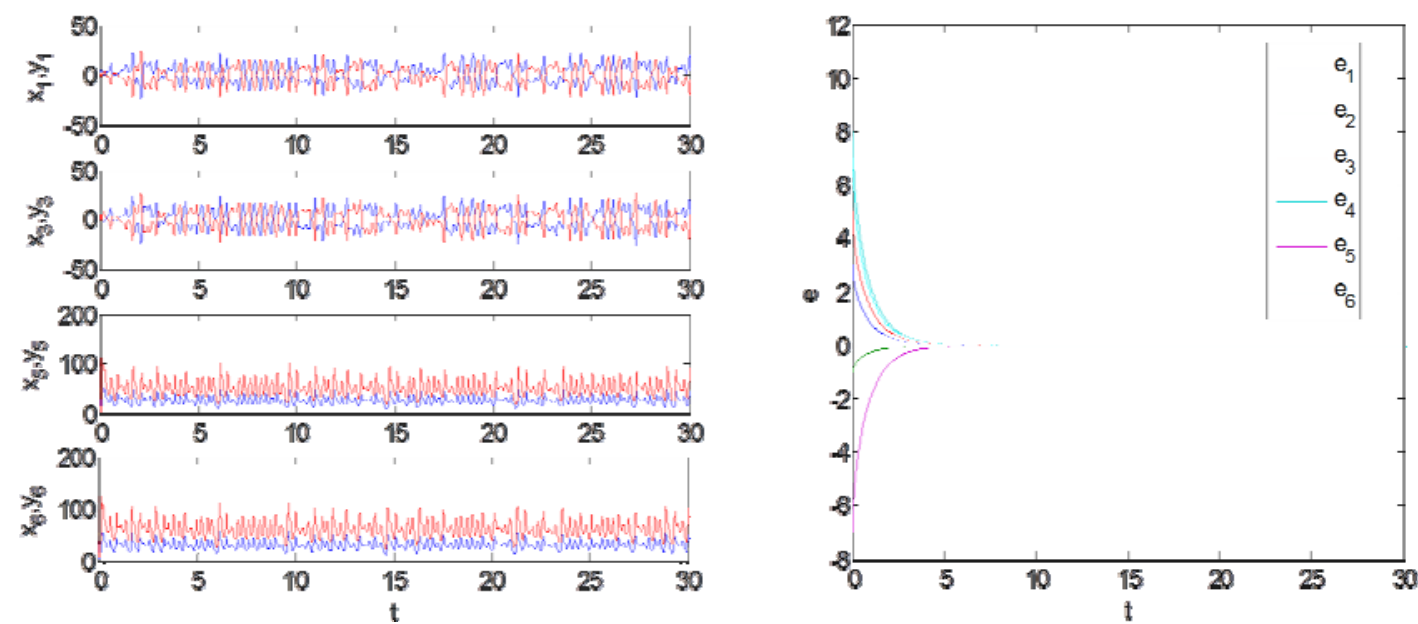

Fig.2. State variables and synchronization errors of drive system and response system for scale factors $m_{1}=-1, m_{2}=-1, m_{3}=2, m_{4}=2$

\section{Conclusions}

In this paper, dynamical behavior of complex system is studied. Based on the stability theory of nonlinear system and control method, the scheme of synchronzaition of the fractional-order hyper-chaotic complex system is designed. Numerical simulations are used to demonstrate the effectiveness and feasibility of the proposed scheme. 


\section{References}

[1] L.M. Pecora, T.L. Carrol: Synchronization in chaotic systems. Phys. Rev. Lett. 64(1990) 821-826.

[2] G.M. Mahmoud, M.A. Al-Kashif, A.A. Farghaly: Chaotic and hyperchaotic attractors of a complex nonlinear system. J. Phys. A: Math. Theor. 41(5), (2008) 055104

[3] P. Seliger, S.C.Young, L.S. Tsimring: Plasticity and learning in a network of coupled phase oscillators. Phys. Rev. E 65(2002) 041906.

[4] R. Carareto, M. S. Baptista, C. Grebogi: Natural synchronization in power-grids with anti-correlated units 18 (2013) 1035-1046

[5]J.H. Peng, E.J. Ding, M. Ding: Synchronizing hyperchaos with a scalar transmitted signal, Phys. Rev. Lett. 76 (1996) 904-907.

[6] Y. Tang, J. Fang: General methods for modified projective synchronization of hyperchaotic systems with known or unknown parameters, Phys. Lett. A . 372 (2008) 1816-1826. 